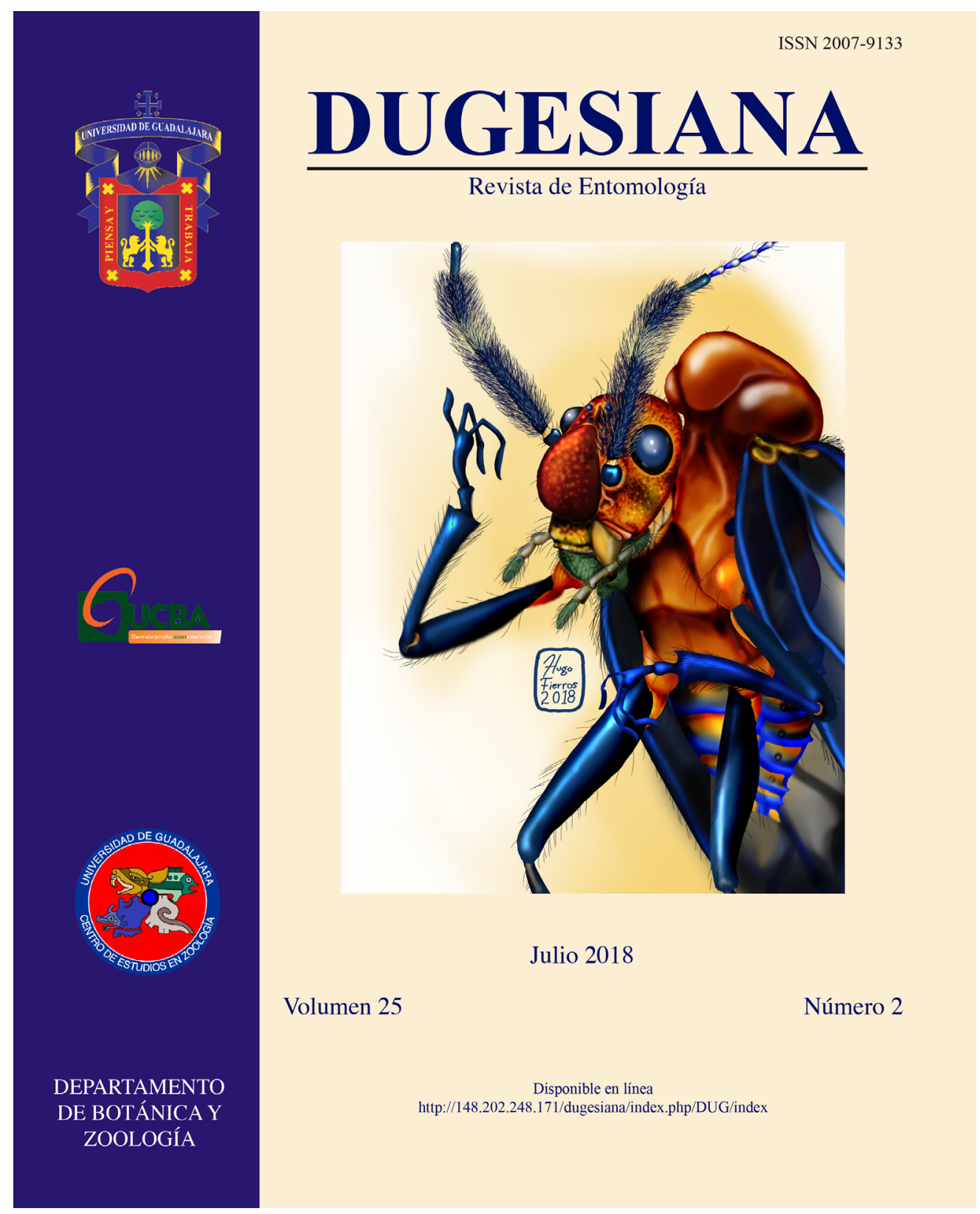

Dugesiana, Año 25, No. 2, julio 2018-diciembre 2018 (segundo semestre de 2018), es una publicación semestral, editada por la Universidad de Guadalajara, a través del Centro de Estudios en Zoología, por el Centro Universitario de Ciencias Biológicas y Agropecuarias. Camino Ramón Padilla Sánchez \# 2100, Nextipac, Zapopan, Jalisco, Tel. 37771150 ext. 33218, http://148.202.248.171/dugesiana/index.php/DUG/index, glenusmx@gmail.com. Editor responsable: José Luis Navarrete Heredia. Reserva de Derechos al Uso Exclusivo 04-2009-062310115100203, ISSN: 2007-9133, otorgados por el Instituto Nacional del Derecho de Autor. Responsable de la última actualización de este número: José Luis Navarrete Heredia, Editor y Ana Laura González-Hernández, Asistente Editorial. Fecha de la última modificación 25 de julio 2018, con un tiraje de un ejemplar.

Las opiniones expresadas por los autores no necesariamente reflejan la postura del editor de la publicación.

Queda estrictamente prohibida la reproducción total o parcial de los contenidos e imágenes de la publicación sin previa autorización de la Universidad de Guadalajara. 


\title{
Primer registro de Laetilia coccidivora (Lepidoptera: Pyralidae) como depredador de Diaspis echinocacti (Hemiptera: Diaspididae) en Tlalnepantla, Morelos \\ First record of Laetilia coccidivora (Lepidoptera: Pyralidae) as predator of Diaspis echinocacti (Hemiptera:
}

Diaspididae) in Tlalnepantla, Morelos

\author{
Juan Manuel Vanegas-Rico ${ }^{1 *}$, J. Refugio Lomeli-Flores ${ }^{1}$, Esteban Rodríguez-Leyva ${ }^{1}$, Jorge Manuel \\ Valdez-Carrasco ${ }^{1}$, Alfonso Luna-Cruz ${ }^{2}$ \\ 'Posgrado en Fitosanidad Entomología y Acarología, Colegio de Postgraduados.. Km. 36.5 Carretera México- \\ Texcoco, Montecillo, 56230 Texcoco, Estado de México, México *autor para correspondencia entomologo. \\ mexicano@gmail.com; ${ }^{2}$ Catedrático CONACYT-Institución de Comisión. Universidad Michoacana de San Nicolás \\ de Hidalgo. Prolongación Mariano Jiménez S/N, Colonia El Varillero, C.P. 60600. Apatzingán, Michoacán, México
}

El pirálido Laetilia coccidivora (Comstock, 1879) (Lepidoptera: Pyralidae) es un depredador de insectos sésiles o de poco movimiento de las familias Coccidae, Eriococcidae, Dactylopiidae y Pseudococcidae (Williams 1993, Solis 2008). En México, L. coccidivora se registra como un insecto benéfico en cultivos de nopal tunero Opuntia megacantha Salm-Dyck 1834 (Cruz-Rodríguez et al. 2016) y nopal verdura Opuntia ficus-indica (Linnaeus) Miller 1768 (Aldama-Aguilera et al. 2005, Portillo y Vigueras 2006, Vanegas-Rico et al. 2010). La producción de $O$. ficus-indica se ha extendido a diversas regiones del mundo para distintos propósitos, mientras que en la república mexicana, su principal productor y consumidor, se ocupan 12,600 ha para verdura y se obtiene una derrama económica de 1.5 mil millones de pesos anuales (SIAP 2015). La producción de estas cactáceas puede afectarse por presencia de insectos escama, algunas de ellas reconocidas como plagas primarias. Debido a la importancia del nopal en México, el presente estudio tuvo como objetivo reportar las especies de escamas plaga que son presas de L. coccidivora en los cultivos de nopal verdura.

Los muestreos se realizaron en Tlalnepantla, Morelos $\left(18^{\circ} 57^{\prime} \mathrm{N}, 98^{\circ} 14^{\prime} \mathrm{O}\right.$, altitud de 1740 y $2500 \mathrm{~m}$ ), la segunda región productora de nopal verdura de México (SIAP 2015). El periodo de muestreo fue de febrero de 2008 a febrero de 2009 , con una frecuencia quincenal. En la zona de estudio se ubicaron cinco sitios de recolecta y en cada uno se seleccionaron al azar diez plantas para búsqueda de plagas (adultos) y del depredador (larvas y pupas). El material se aisló en cajas Petri de plástico, manteniendo los individuos inmaduros en condiciones controladas $\left(25 \pm 2{ }^{\circ} \mathrm{C}\right.$, HR $60 \pm 10$ $\%$, fotoperiodo 12:12). Algunos ejemplares de escamas se montaron de acuerdo a la técnica de Hamon y Kosztarab (1979) para su determinación con las claves de De Lotto (1974) y Gill (1997). Los adultos de L. coccidivora se compararon con ejemplares de las colecciones del Colegio de Postgraduados y la colección personal del primer autor.

Como resultado, se determinaron dos presas de L. coccidivora en nopales de Tlalnepantla, Morelos:
Dactylopius opuntiae (Cockerell, 1896) y Diaspis echinocacti (Bouché, 1833) de las familias Dactylopiidae y Diaspididae respectivamente. La abundancia anual de $L$. coccidivora fue 1,801 individuos totales sobre colonias de D. opuntiae, mientras que en Di. echinocacti se obtuvieron sólo cinco individuos totales y ocurrió durante mayo de 2008. Estos últimos se recolectaron en etapa de larva y con el pupario desarrollado, de los cuales emergieron dos ejemplares en laboratorio.

D. opuntiae se asocia a cactáceas platiopuntias, principalmente Opuntia spp. (Chávez-Moreno et al. 2009, García et al. 2016) y se reconoce como una plaga primaria del cultivo de O. ficus-indica en Tlalnepantla, Morelos (Vanegas-Rico et al. 2010, 2017) y otras regiones del mundo (García et al. 2016). Mientras que Di. echinocacti se asocia mayormente a cactáceas (40 géneros) pero también tiene hospederos de otras familias de plantas (Lima y Gama 2001, García et al. 2016); y aunque se cita como una plaga relevante en cultivos de nopal (Russo y Siscaro 1994, Miller y Davidson 2005, De Souza et al. 2009), en Tlalnepantla no se considera una plaga, ya que se recolectó en menos de seis plantas/ha y presentó una infestación menor al 15\% del área del cladodio. Es probable que exista un control natural por parasitoides sobre Di. echinocacti, puesto que se observaron orificios de emergencia de micro himenópteros. Respecto a depredadores, aunque se registran siete especies alimentándose de D. opuntiae (Vanegas-Rico et al. 2010) sólo L. coccidivora depredó a Di. echinocacti y desarrolló sus puparios con restos de sus presas, pero sin cubrir más del $20 \%$ del pupario o incluso sin restos de presas (Fig. 1b). En contraste, los individuos de L. coccidivora recolectados en D. opuntiae se desarrollaron debajo de sus colonias y utilizaron sus restos para cubrir completamente sus puparios (Fig. 1a), esta conducta también la realiza con otros insectos sésiles (Williams 1993).

La diferencia en abundancia de L. coccidivora ante estas dos distintas presas probablemente se debió a que Di. echinocacti fue un recurso escaso y nutrimentalmente insuficiente para soportar altas poblaciones de este 
depredador. Esta afirmación se basa en la correlación positiva del pirálido $(r=0.40, p=0.00)$ respecto al aumento de hembras/colonia de D. opuntiae (Vanegas-Rico et al. 2017) y su presencia recurrente en densidades superiores a 100 colonias de cochinilla de nopal por cladodio (CruzRodríguez et al. 2016). La voracidad de este depredador está en proceso de cuantificación (Rodríguez-Leyva comunicación personal), pero las observaciones personales sugieren que un individuo de L. coccidivora consume varias colonias de $D$. opuntiae durante su etapa larval; y en caso de faltar alimento, puede recurrir al canibalismo o a depredar otros enemigos naturales. Aunque las colonias de Di. echinocacti eran maduras, su densidad poblacional fue baja y sólo se registró en el mes de mayo, periodo en el que inicia el aumento poblacional de L. coccidivora en México (Vanegas-Rico et al. 2017) y Estado Unidos (Powell 1980). Finalmente, este es el primer registro de L. coccidivora como depredador de Di. echinocacti, la cual probablemente es una presa alterna en los cultivos de nopal verdura de Tlalnepantla Morelos.

\section{AGRADECIMIENTOS}

El primer autor agradece a CONACYT por la beca completa para estudios de Maestría, a COMECYT por el apoyo económico durante la escritura de tesis de maestría. Al Consejo Municipal de Nopaleros de Tlalnepantla (COMUNOTLA) y al Grupo Interdisciplinario de Investigación de Nopal (GIIN) por el apoyo brindado en campo. El último autor agradece la oportunidad de Catedrático CONACYT.

\section{LITERATURA CITADA}

Aldama-Aguilera, C., C. Llanderal-Cázares, M. SotoHernández and L. E. Castillo-Márquez. 2005. Cochineal (Dactylopius coccus Costa) production in prickly pear plants in the open and in microtunnel greenhouses. Agrociencia 39(2): 161-171.

Chávez-Moreno, C. K., A. Tecante, and A. Casas. 2009. The Opuntia (Cactaceae) and Dactylopius (Hemiptera: Dactylopiidae) in Mexico: a historical perspective of use, interaction and distribution. Biodiversity and Conservation, 18(13): 3337-3355.

Cruz-Rodríguez J. A., E. González-Machorro, A. A. Villegas González, M. L. Rodríguez Ramírez, and F. Mejía Lara. 2016. Autonomous biological control of Dactylopius opuntiae (Hemiptera: Dactylopiidae) in a prickly pear plantation with ecological management. Environmental Entomology, 45 (3): 642-648.

De Lotto, G. 1974. On the status of the cochineal insects (Homoptera: Coccoidea: Dactylopiidae). Journal of Entomological Society of South Africa, 37: 167-193.

De Souza, F., M. J. Cerqueira, H. Monaísy, V. de Melo, M. Forti, and D. M. Passos. 2009. Control of Diaspis echinocacti (Bouché, 1833) (Hemiptera: Diaspididae). In: Prickly-Pear. In: Proceedings of the VI International Congress on cactus and Cochineal. Acta Horticulturae,
(811): 223-226.

García, M, B. D. Denno, D. R. Miller, G. L. Miller, Y. Ben-Dov, and N.B. Hardy. 2016. ScaleNet: A literaturebased model of scale insect biology and systematics. Database. http://scalenet.info. Consultado el $25 \mathrm{de}$ enero de 2017.

Gill, R. J. 1997 The Scale Insects of California: Part 3. The Armored Scales (Homoptera: Diaspididae). California Department of Food \& Agriculture Sacramento, CA 307 p.

Hamon, A. B. and M. L. Kosztarab. 1979. Morphology and systematic of the first instar of the genus Cerococcus (Homoptera: Coccoidea: Cerococcidae). Virginia Polytechnic Institute and State University. Research Division, (146): 1-122.

Lima, I. M. M. and N. S. Gama. 2001. Registro de plantas hospedeiras (Cactaceae) e de nova forma de disseminação de Diaspis echinocacti (Bouché) (Hemiptera: Diaspididae), Cochonilha-da-PalmaForrageira, nos Estados de Pernambuco e Alagoas. Neotropical Entomology, 30(3): 479-481.

Miller, D. R. and J. A. Davidson. 2005. Armored Scale Insect Pests of Trees and Shrubs. Cornell Univ. Press Ithaca, NY $442 \mathrm{p}$.

Powell, J. A. 1980. Five insects believed to be newly established or recorded on Santa Cruz Island, California (Dermaptera, Lepidoptera). Bulletin of Southern California Academic of Science, 79(3): 97-108.

Portillo, L. and A. L. Vigueras. 2006. A review on the cochineal species in México, host and natural enemies. Acta Horticulturae, 728: 249-255.

Russo, A. and Siscaro, G. 1994 Diaspis echinocacti fitomizo del fico d'India in Sicilia. Informatore Agrario, 50(37): 73-76.

SIAP. Servicio de Información Agroalimentaria y Pesquera Cierre de producción anual 2015. http://infosiap. siap.gob.mx/aagricola_siap_gb/ientidad/index.jsp. Consultado el 05 de abril de 2017.

Solis, M. A. 2008. Pyraloidea and Their Known Hosts (Insecta: Lepidoptera) of Plummers Island, Maryland. Bulletin of the Biological Society of Washington, 15(1): 88-106.

Vanegas-Rico J. M., J. R. Lomeli-Flores, E. RodríguezLeyva, G. Mora-Aguilera y J. Valdez. 2010. Enemigos naturales de Dactylopius opuntiae (Cockerell) en Opuntia ficus-indica (L.) Miller en el centro de México. Acta Zoológica Mexicana (n.s.), 26(2): 415-433.

Vanegas-Rico, J. M., A. Pérez-Panduro, J. R. Lomeli-Flores, E. Rodríguez-Leyva, J. M. Valdez, G. Mora-Aguilera. 2017 - Dactylopius opuntiae (Hemiptera:Dactylopiidae) population fluctuations and predators in Tlalnepantla, Morelos. Folia Entomológica Mexicana 3(2):23-31.

Williams M. L. 1993. Toumeyella lignumvitae, a new species of scale insect from the Florida keys (Homoptera: Coccidae). Florida Entomologist, 76(4): 566-572. 
Recibido: 26 de abril 2017

Aceptado: 1 de diciembre 2017

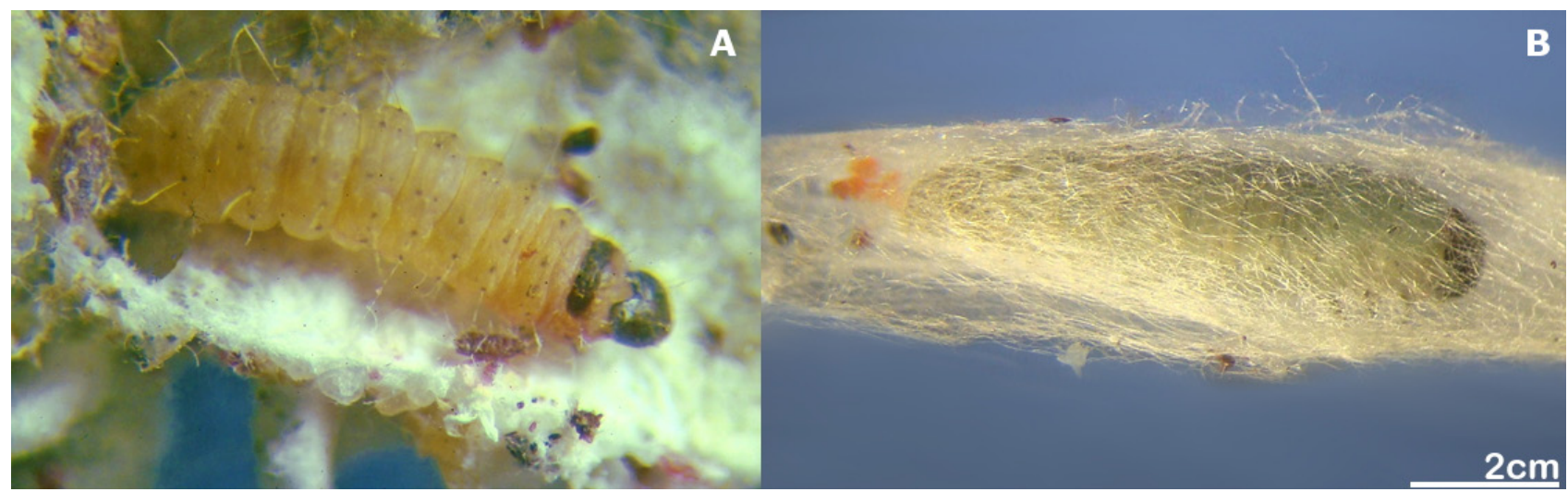

Figura 1. Comparación de puparios en larvas de Laetilia coccidivora alimentándose sobre dos especies de escamas de nopal. a) pupario con restos de Dactylopius opuntiae, b) pupario sin restos de Diaspis echinocacti. 